\title{
Thermoelastic analysis of a copper-based monocristalline shape-memory alloy
}

\author{
D. Delpueyo ${ }^{1}$, X. Balandraud ${ }^{1, a}$, M. Grédiac ${ }^{1}$ and R.Y. Fillit ${ }^{2}$ \\ ${ }^{1}$ Laboratoire de Mécanique et Ingénieries (LaMI), Université Blaise Pascal (UBP) \& Institut \\ Français de Mécanique Avancée (IFMA), Campus de Clermont-ferrand - Les Cézeaux, BP 265, \\ 63175 Aubière cedex, France \\ 2 École Supérieure des Mines de Saint-Étienne, Centre « Science des Matériaux et des Structures » \\ UMR CNRS 5176 PECM, 158 cours Fauriel, 42023 Saint-Etienne cedex 2, France.
}

\begin{abstract}
The objective of this study is to observe the thermoelastic coupling effect in a monocrystalline $\mathrm{Cu}-\mathrm{Al}-\mathrm{Be}$ shape-memory alloy. The orientation of the austenite crystal is measured by Microfocus X-ray texture analysis. Uniaxial cyclic loading is applied to the specimen at room temperature. Infrared thermography is used to capture temperature fields on the specimen surface. The approach consists in analysing the cyclic temperature oscillation of points located on the surface of the specimen. The study highlights first the significant difference between the thermoelastic couplings in austenite and martensite. Second the anisotropy of both phases, especially the martensite, is evidenced.
\end{abstract}

\section{Introduction}

Shape memory alloys (SMAs) exhibit peculiar properties due to austenite-martensite transformations triggered by stress and temperature. Thermomechanical couplings play an important role in the response of the material under mechanical load, even at constant room temperature [1-12]. Some other thermal effects are related to the thermoelastic coupling, also named isentropic coupling, which is the coupling between the temperature and the elastic part of the strain through the coefficient of thermal expansion (CTE) appearing in the free energy expression [13]. The so-called thermoelastic stress analysis, based on this coupling, enables to measure stresses in a structure subjected to cyclic loading. Although the technique is widely applied to many materials, very few articles present applications to SMAs [14-17] to the best knowledge of the authors.

In the present study, infrared (IR) thermography is used to capture temperature fields on a monocrystalline copper-aluminium-beryllium (Cu-Al-Be) SMA subjected to a mechanical load at room temperature. The objective is to distinguish the two different phases by observing the thermoelastic coupling effect.

Section 2 describes the specimen under study and the experimental set-up. Section 3 presents the data post-processing of the temperature maps provided by the IR camera. Experimental results are discussed in Section 4.

\footnotetext{
a e-mail : xavier.balandraud@ifma.fr
} 


\section{Experimental set-up}

\subsection{Specimen}

The specimen is a $18 \times 70 \times 1 \mathrm{~mm}^{3}$ rectangular sheet along the $\mathbf{x}$-, $\mathbf{y}$ - and $\mathbf{z}$-directions, respectively. It is made of a monocrystalline $\mathrm{Cu}-\mathrm{Al}-\mathrm{Be}$ SMA. Specimen manufacturing (by Nimesis Technology, Metz, France) is based on the following procedure: casting of polycrystalline bulk at the desired composition; transformation to single crystal by vertical Bridgman method; solution heat treatment between $800^{\circ} \mathrm{C}$ and $850^{\circ} \mathrm{C}$ (austenisation) and quenching to keep the austenitic state at room temperature; electro-erosion machining to obtain specimen with desired dimensions. The specimen is austenitic at room temperature. Aluminium tabs are bonded on the two ends of the specimen in order to protect it once placed in the grips of the uniaxial testing machine. The gauge length is equal to $45 \mathrm{~mm}$.

$\mathrm{X}$-ray diffraction technique is used as macrotexture measurement. Progress in nanotechnologies, leading to enhanced precision in machining microsystems, now transforms X-ray diffraction into Xray microfocus measurement (Figure 1). The irradiated spot, basically a circle which undergoes distortions when the sample is tilted and rotated, integrates the texture over a surface of about 2 $\mathrm{mm}^{2}$. The X-PERT MRD equipment [18-19] for texture analysis consists of a X-ray tube, a goniometer, a detector and subsequent treatment. The sample holder with 3 dimension movements and excellent positioning is required when microfocus is used. Because of the extreme precision required in the positioning, the $\mathrm{X}$-ray source must be emitted through $\mathrm{X}$-ray lenses acting as wave guides, so that the beam is almost perfectly parallel. On the specimen, $\{220\}$ and $\{400\}$ pole figures are measured (Figure 2). The rotation angle $\Phi$ is varied from 0 to $360^{\circ}$ by steps of $1^{\circ}$ and the tilt angle $\psi$ is varied up to $85^{\circ}$ by steps of $1^{\circ}$. Due to thermal treatments, the specimen surface is slightly oxidised; so stresses and strains exist at the specimen surface; nevertheless, X-ray measurement gives a cubic crystal structure Pm3m with lattice parameter $a_{0}=0.58157 \mathrm{~nm}$. The crystal orientation with respect to the specimen axis is nearly a Goss $\{011\}<100>$ texture with the (011) plane parallel to the specimen surface $(\mathbf{x}, \mathbf{y})$ and the $<100>$ crystallographic direction parallel to the $\mathbf{y}$-axis. The miss orientation is nearly a $3^{\circ}$ tilt of the $(011)$ plane around the $\mathbf{x}$-axis.

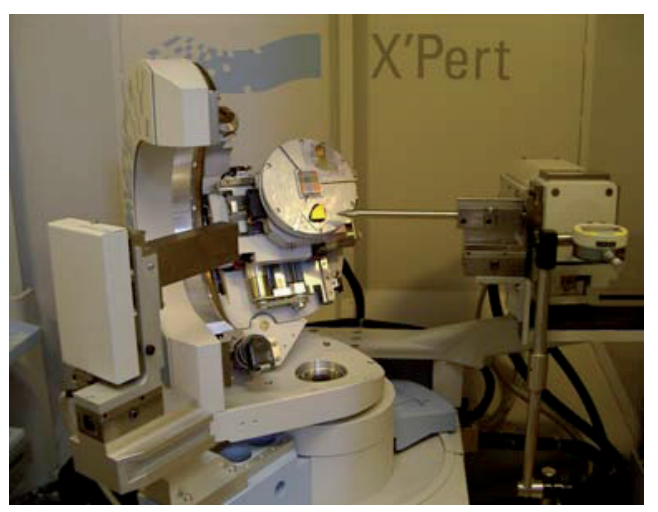

Fig. 1. Equipment for crystal orientation measurement 

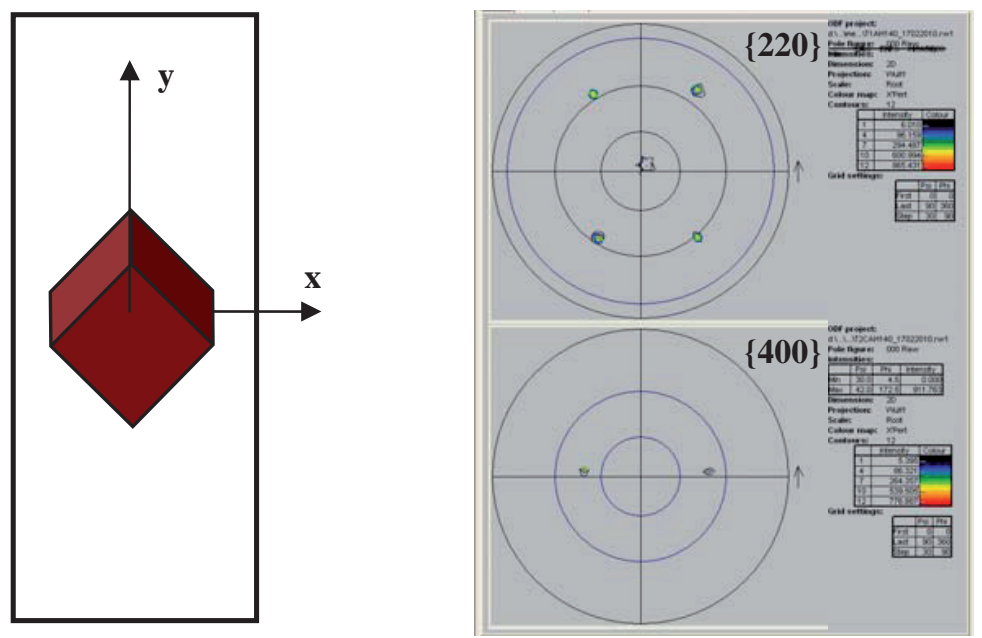

Fig. 2. Measurement of the austenite crystal orientation

\subsection{Mechanical loading}

Mechanical tests are performed with a uniaxial testing machine MTS $(+/-15 \mathrm{kN})$ at room temperature. The loading process consists first of a quasi-static displacement imposed to the moving grip in order to partially transform the specimen to martensite. Then an oscillation around the displacement reached in the first step is applied to the specimen. Several values of macroscopic strain $\varepsilon_{\text {macro }}$ are successively applied for a given test. The objective of the thermal analysis during the oscillating steps is to observe the thermoelastic coupling effect as a function of $\varepsilon_{\text {macro }}$.

\subsection{Temperature measurement}

A Cedip Jade III-MWIR infrared camera is used to capture the temperature fields on the specimen surface. This camera features IR detectors with a wavelength range of 3.5-5 $\mu \mathrm{m}$. The thermal resolution at room temperature is about $20 \times 10^{-3} \mathrm{~K}$. The integration time used for the measurement is $1500 \mu \mathrm{s}$. The acquisition frequency is $f_{a}=436 \mathrm{~Hz}$. It is noted that this acquisition frequency $f_{a}$ is not a multiple of the load frequency $f_{L}$ for a better data processing by discrete Fourier transform. The maps actually provided by the camera are composed of $80 \times 160$ pixels. The spatial resolution, corresponding here to the pixel size on the specimen, is equal to $0.49 \mathrm{~mm}$.

A thin, opaque and uniform black paint is spayed on the specimen surface to obtain a thermal emissivity close to one. It must be pointed out that this paint spray is applied only a few minutes before testing the specimen. The reason is the fact that paint becomes then more brittle, so small cracks in the paint layer would be observed for the highest strain levels and those cracks would disturb temperature measurement.

\section{Data post-processing}

\subsection{Thermomechanical framework}

The heat diffusion equation is written (see Ref. [1,2,5-12] for more details):

$$
\rho C \partial T / \partial t-\underline{k} \operatorname{div} \operatorname{grad} T=s+r_{e x t}
$$


where $\rho$ is the density, $C$ the heat capacity, $T$ the temperature, $\underline{k}$ the second-order thermal conductivity tensor, $r_{e x t}$ the external heat source by radiation and $s$ the heat source produced by the material itself. In the present experiments, the heat source $s$ can be split in two terms:

- $\quad$ the thermoelastic coupling $s_{\text {the }}$, also called isentropic coupling. It can be written as follows:

$$
s_{\text {the }}=-T \mathbb{A} \underline{\alpha}: \partial \underline{\varepsilon}^{e l} / \partial t
$$

where $\mathbb{A}$ is the fourth-order stiffness tensor and $\underline{\alpha}$ the second-order thermal expansion tensor. $\underline{M}: \underline{N}$ is the trace of the matrix product $\underline{M} \times \underline{N}$;

- $\quad$ the mechanical dissipation $d_{l}$, also called intrinsic dissipation. This term is always positive. It is related to some irreversible phenomena such as fatigue damage.

A data processing of the thermal data is performed during oscillating steps described in Section 2.2. If no latent heat is produced and adiabatic conditions are achieved, integrating Equation (1) over one half cycle leads to:

$$
\rho C \Delta T=T_{0} \Delta\left(\mathbb{A} \underline{\alpha}: \underline{\varepsilon}^{e l}\right)
$$

where $\Delta T$ is the amplitude of temperature oscillation, $\Delta\left(\mathbb{A} \underline{\alpha}: \underline{\varepsilon}^{e l}\right)$ the amplitude of $\mathbb{A} \underline{\alpha}: \underline{\varepsilon}^{e l}$ and $T_{0}$ the mean temperature in Kelvin.

\subsection{Calculation of amplitude during oscillating steps}

As expected, experimental results exhibit temperature oscillations at the same frequency as the load during oscillating steps. A Fourier analysis can be performed because of the periodic nature of the signal. The amplitude $\Delta T$ of the temperature oscillation is obtained for each pixel. The value is then normalized by the macroscopic uniaxial stress amplitude $\Delta \sigma_{\text {macro }}$ :

$$
\text { Normalised temperature amplitude }=\Delta T / \Delta \sigma_{\text {macro }}
$$

With isotropic materials, Equation (3) shows that the normalised temperature amplitude would be equal to $\alpha T_{0} / \rho C$; so its value would be specific to the considered material and phase.

\subsection{Remark about anisotropy}

Austenite phase has anisotropic properties [20,21]. So the thermoelastic coupling is expected to be dependent on the unstressed single crystal orientation with respect to the load axis $\mathbf{y}$. Second, the martensite phase is also anisotropic; moreover it exists through different variants due to the symmetry breaking occurring in the phase change. These variants have the same crystal structure, but are rotated through different angles in space with respect to the parent phase. Consequently, the thermoelastic coupling is also expected to be strongly dependent on the orientation of each martensite variant produced in the specimen.

\section{Experimental results}

\subsection{Influence of the macroscopic strain $\varepsilon_{\text {macro }}$}

Figure 3 presents three maps of normalised temperature amplitude $\Delta T(x, y) / \Delta \sigma_{\text {macro }}$ as a function of macroscopic strain $\varepsilon_{\text {macro }}$ :

- $\quad$ For a purely austenitic specimen (Figure 3.a), the thermal signal is very small. Increasing the temperature oscillation amplitude would be possible by increasing the load amplitude. 
However, it would create a cyclic austenite-martensite transformation: latent heat would be cyclically absorbed and released. This would not suit the objective of the present study.

- Figure 3.b shows strong concentrations of $\Delta T / \Delta \sigma_{\text {macro }}$ in a partially transformed specimen. These concentrations consist of bands. Most of these bands are parallel and inclined with respect to the load axis $\mathbf{y}$. The band angles can be compared with the orientations of the habit-planes predicted from the compatibility condition between austenite and martensite. It can be deduced from Equation (3) that the temperature oscillation on a martensitic zone strongly depends on the local elastic strain $\underline{\varepsilon}^{\text {el }}$ and/or on the local martensite variant through the anisotropy of its tensor $\mathbb{A} \underline{\alpha}$.

- When the specimen approaches to the quasi purely martensite state (Figure 3.c), the heterogeneity of $\Delta T / \Delta \sigma_{\text {macro }}$ decreases: the bottom part of the specimen still exhibits a strong concentration, while most of the specimen presents a reduced heterogeneity compared to the cases with lower macroscopic strains $\varepsilon_{\text {macro }}$. However, the heterogeneities in the blue zones are actually captured by the data processing: the heterogeneity in these zones can be evidenced by a change in the color scale.

As a conclusion, it can be noted that the thermoelastic coupling in austenite may be quite difficult to observe, contrary to martensitic zones which reveal clear temperature oscillations due to thermoelastic coupling.

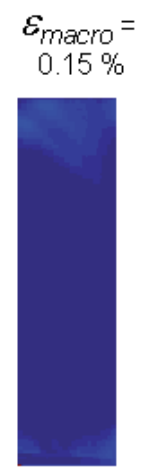

a)

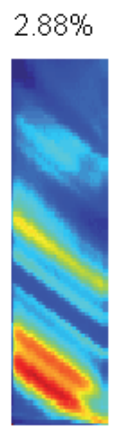

b)

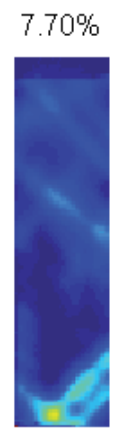

c)

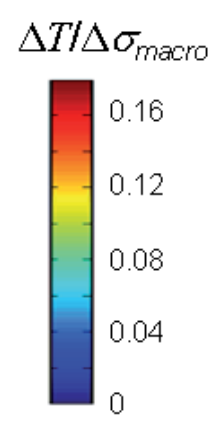

Fig. 3. Normalised temperature amplitude $\Delta T / \Delta \sigma_{\text {macro }}$ as a function of the macroscopic strain $\varepsilon_{\text {macro }}$ with $\Delta \varepsilon_{\text {macro }}$ fixed to $\pm 0.096 \%$ : for the specimen which is purely austenitic $\left(\varepsilon_{\text {macro }}=0.15 \%\right)$, partially transformed $\left(\varepsilon_{\text {macro }}=2.88 \%\right)$ and quasi purely martensitic $\left(\varepsilon_{\text {macro }}=7.69 \%\right)$

\subsection{Influence of the strain amplitude $\Delta \varepsilon_{\text {macro }}$}

Figure 4 presents the results obtained with different strain oscillation amplitudes $\Delta \varepsilon_{\text {macro }}$. For a value of $\varepsilon_{\text {macro }}$ fixed at $3.85 \%$, different stress amplitudes $\Delta \sigma_{\text {macro }}$ are obtained by varying the value of $\Delta \varepsilon_{\text {macro }}$. Two small zones are selected in the specimen. The first one is supposed to be purely austenitic since the corresponding temperature oscillation is small (see Section 4.1 above). The second one is located in a concentration zone of the $\Delta T / \Delta \sigma_{\text {macro }}$ ratio. Figure 4 presents the temperature oscillation amplitude $\Delta T$ as a function of $\Delta \sigma_{\text {macro }}$ for the two zones. According to Equation (3), the curves should be linear with slopes depending on the orientation of the crystal (martensite variant or austenite). The curves obtained are indeed quite linear. Another remark is that the amplitude of the thermal response of austenite is confirmed to be very low compared to the thermal response of martensite. 


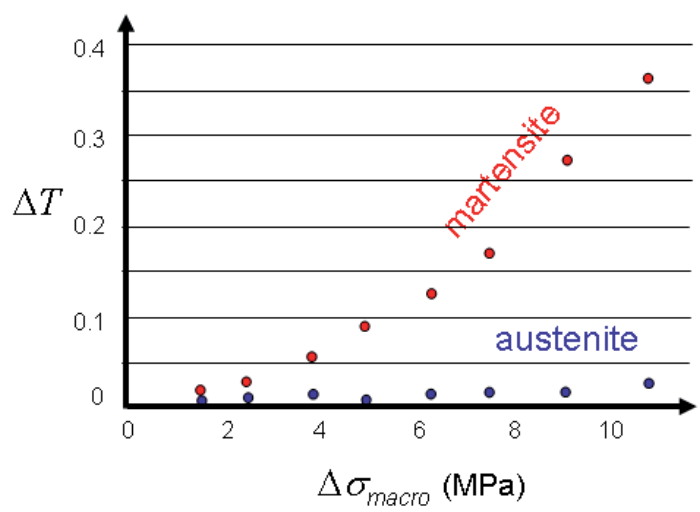

Fig. 4. Temperature oscillation amplitude $\Delta T$ as a function of the stress amplitude $\Delta \sigma_{\text {macro }}$, for a fixed value of the strain $\varepsilon_{\text {macro }}$ at $3.85 \%$.

\section{Conclusion}

The objective of this study was to observe the thermoelastic coupling effect in a monocrystalline $\mathrm{Cu}-\mathrm{Al}-\mathrm{Be}$ shape-memory alloy. At room temperature, infrared thermography was used to capture temperature fields on the surface of the specimen subjected to slight cyclic loadings around macroscopic strains previously applied. Martensitic microstructures mainly consisting of parallel bands are revealed by the analysis, although other microstructure types have been also observed. The lack of information in the literature about the anisotropy of the thermal expansion tensor in SMA crystals is underlined by the authors. The present experiments show first that a large difference between the thermoelastic couplings of austenite and martensite phases exists. Second, the effect of crystal anisotropy has been emphasized, especially for the martensite phase.

\section{References}

1. A. Chrysochoos, H. Pham, O. Maisonneuve C. R. Acad. Sc. série II 316, 1031 (1993)

2. J.A. Shaw, S. Kyriakides, J. Mech. Phys. Solids 43, 1243 (1995)

3. A. Chrysochoos, H. Pham, O. Maisonneuve, Nuclear Eng. Design 162, 1 (1996)

4. J.A. Shaw, S. Kyriakides, Acta Mat. 45, 683 (1997)

5. R. Peyroux, A. Chrysochoos, C. Licht, M. Löbel, Int. J. Eng. Sc. 36, 489 (1998)

6. X. Balandraud, E. Ernst, E. Soos, C.R. Acad. S. - série IIb - Mécanique 327, 33 (1999)

7. X. Balandraud, E. Ernst, E. Soos, Z. Angew. Math. Phys. 51, 419 (2000)

8. X. Balandraud, A. Chrysochoos, S. Leclercq, R. Peyroux, C. R. Acad. Sc. série IIb Mécanique 329, 621 (2001)

9. A. Chrysochoos, C. Licht, R. Peyroux, C. R. Mécanique 331, 25 (2003)

10. N. Chaillet, C. Lexcellent, J. Abadie, C. R. Mécanique 332, 147 (2004)

11. P. Schlosser, H. Louche, D. Favier, L Orgeas, Strain 43, 260 (2007)

12. P. Schlosser, D. Favier, H. Louche, L. Orgeas, State-of-art research and application of SMAs technology, Book Series: Advances in Science and Technology, 59, 140 (2009)

13. P. Stanley, Strain 44, 285 (2008)

14. J. Eaton-Evans, J.M. Dulieu-Barton, E. Little, I. Brown, Advances in Experimental Mechanics IV, Book Series: Applied Mechanics and Materials 3-4, 47 (2005)

15. J. Eaton-Evans, J.M. Dulieu-Barton, E.G. Little EG, I.A. Brown, J. Strain Analysis Eng. Design 41, 481 (2006) 
16. J. Eaton-Evans, J.M. Dulieu-Barton, E.G. Little, I.A. Brown, Advances in Experimental Mechanics VI, Book Series: Applied Mechanics and Materials 13-14, 225 (2008)

17. J. Eaton-Evans, J.M. Dulieu-Barton, E.G. Little, I.A. Brown IA, Proceedings of the Institution of Mechanics Engineers Part C - Journal of Mechanical Engineering Science 222, 97(2008)

18. J.Y. Poussardin, PhD dissertation, Ecole des Mines de Saint-Etienne, France (2003)

19. M. Darrieulat, K. Zhani, R.Y. Fillit, A. Chenaoui, J. Eng. Mat. Tech. 131, 1 (2009)

20. V. Novak, P. Sittner, D. Vokoun, N. Zarubova, Materials Sc. and Eng. A 273, 280 (1999)

21. A. Planes, Ll. Manosa, Solid State Physics 55, 159 (2001) 